\title{
Contraception for women with epilepsy: counseling, choices, and concerns
}

\author{
This article was published in the following Dove Press journal: \\ Open Access Journal of Contraception \\ 19 April 2016 \\ Number of times this article has been viewed
}

\begin{abstract}
Arne Reimers ${ }^{1,2}$
'Department of Clinical Pharmacology, St Olavs University Hospital, ${ }^{2}$ Department of Laboratory Medicine, Children's and Women's Health, Faculty of Medicine, Norwegian University of Science and Technology (NTNU), Trondheim, Norway
\end{abstract}

Abstract: Approximately 50\% of all pregnancies in women with epilepsy (WWE) occur unplanned. This is worrying, given the increased occurrence of obstetrical complications in WWE, including the risk of seizures and their possible consequences for both the mother and the unborn child. Hormonal contraception is usually regarded as highly effective, but it is subject to numerous bidirectional drug interactions with several antiepileptic drugs. These interactions may lead to loss of seizure control or contraceptive failure. Further concerns are loss of bone mineral density and increased seizure activity due to hormonal effects. Many physicians lack sufficient knowledge regarding these issues, and most WWE have never received adequate counseling. Moreover, several studies show that a large proportion of WWE do not take their medicines regularly. This article reviews all of these issues and offers practical recommendations for the management of contraception in WWE.

Keywords: ethinyl estradiol, progestins, epilepsy, antiepileptic drugs, interactions, pregnancy, seizure aggravation, bone mineral density

\section{Introduction}

With an estimated prevalence of $0.3 \%-0.7 \%$, epilepsy is one of the most frequent neurological diseases. ${ }^{1}$ Women with epilepsy (WWE), like healthy women, use different kinds of contraceptive methods, ie, hormonal contraception (HC), intrauterine devices (IUDs), barrier methods, or combinations of them. HC includes combined oral contraceptives (COCs), progestin-only pills, intramuscular injections, subdermal implants, skin patches, hormone-releasing IUDs, and vaginal rings. However, most WWE also use antiepileptic drugs (AEDs). Many of these drugs do interact with HC, which may lead to contraceptive failure or impaired seizure control..$^{2,3}$ Either of these complications may have serious social, psychological, professional, and economic consequences. Additionally, many AEDs possess teratogenic potential and/or may exert a negative impact on cognitive and psychomotor skills of children exposed to these AEDs in the womb. ${ }^{4-6}$

Given the above, it is deeply concerning that $\sim 50 \%$ of all pregnancies among WWE occur unintended. ${ }^{7,8}$ This is about the same proportion as found in the general population. ${ }^{9,10}$ Risk factors for unintended pregnancy include low socioeconomic status, low education, and ethnicity. ${ }^{8}$ Also, only half of all WWE using contraception do so with a highly effective method (HC, IUDs, or surgery). Moreover, many WWE use enzymeinducing AEDs that may impair the efficacy of highly effective $\mathrm{HC}^{8}$

It might be speculated whether better education of WWE could reduce the proportion of unplanned pregnancies. However, several studies show that a large proportion 
of doctors, including neurologists and gynecologists, lack sufficient knowledge about reproductive health issues of WWE and how these may be affected by AEDs. ${ }^{11-13}$ Hence, the current treatment guidelines may not be followed. ${ }^{14}$ More recent surveys found a trend from prescribing older AEDs toward newer AEDs with a more favorable interaction and safety profile, which may indicate a growing awareness among doctors. ${ }^{15-18}$ However, most WWE do not receive necessary information. ${ }^{19,20}$ A 2015 survey found that $<7 \%$ of women received contraceptive counseling. ${ }^{21}$ Even when information is provided, many WWE do not recall the information they were given. ${ }^{22}$ Consequently, most WWE have only limited knowledge about interactions between $\mathrm{HC}$ and AEDs and potentially harmful effects of AEDs on the child. ${ }^{23,24}$

Apart from drug interactions between HC and AEDs, contraception for WWE comprises further questions. Does HC aggravate seizures? Is there an increased risk of osteoporosis? Is adherence an issue? The answers to these questions affect treatment options and need to be discussed with the patient. Hence, the attending physician should have thorough knowledge.

\section{Epilepsy, AEDs, and female fertility}

While many WWE will have normal sex lives and normal pregnancies, they in general have lower fertility rates than healthy women. Also, anovulatory cycles, irregular menstrual bleedings, or oligo-/amenorrhea occur more frequently in WWE than in women without epilepsy. ${ }^{25}$ This is more common with antiepileptic polytherapy than with monotherapy, which suggests a causal role of AEDs. However, available data also suggest that seizures and epileptic discharges, especially temporal lobe epilepsy, may negatively affect ovarian function and ovulation via disturbances in the hypothalamicpituitary axis. ${ }^{25-27}$

It is well established that enzyme-inducing AEDs may increase sex hormone-binding globulin and induce the metabolism of sex steroid hormones, thus reducing the serum concentration of free and total sex hormones. ${ }^{28,29}$

Valproate, one of the most frequently used AEDs, can induce polycystic ovary syndrome. ${ }^{30}$ This syndrome consists of polycystic ovaries, hyperandrogenism, menstrual disturbances, and anovulatory cycles. The underlying mechanisms are not completely understood, but an interaction of valproate with sex steroid synthesis and metabolism in the ovary is presumed. $^{25}$

WWE also have a higher risk for complications during pregnancy and delivery, eg, hypertension, preeclampsia, bleedings, preterm birth, or small for gestational age. The frequency of cesarean section is also higher. ${ }^{31}$

In conclusion, current evidence suggests that both epilepsy itself as well as drug treatment with AEDs may affect female fertility and cause complications during pregnancy and delivery. These findings underline the importance of pregnancy planning, which includes contraception.

\section{What AEDs do to HC}

Many of the "old" or "first-generation" AEDs (phenytoin, phenobarbital, primidone, carbamazepine) and several of the "new" or "second-generation" AEDs (oxcarbazepine, eslicarbazepine, topiramate, felbamate, rufinamide, perampanel) have more or less pronounced enzyme-inducing effects. They may induce either cytochrome P450 (CYP) enzymes, uridine-diphosphate-glucuronosyltransferase (UGT) enzymes, or both, thereby accelerating the metabolism of steroid hormones. Contraceptive failure provoked by enzyme-inducing AEDs is common and may affect both oral and nonoral HC. ${ }^{32-34}$ The estrogen compound used in combined HC usually is ethinyl estradiol (EE), which has been used for decades. EE has a well-known pharmacokinetic and interaction profile. It is mainly metabolized by CYP 3A4, but conjugation by UGT also plays a role. ${ }^{35}$ Besides $\mathrm{EE}$, there is a plethora of older and newer progestins used for HC. ${ }^{36,37}$ Their metabolism and possible interactions with AEDs are much less studied. In general, their metabolism is inducible like that of EE. Thus, their contraceptive effect may fail when they are coadministered with carbamazepine or other enzyme-inducing AEDs. Examples for this include oral levonorgestrel, oral norethindrone, and the subdermal etonogestrel implant. ${ }^{32,33,38,39}$ The interaction potential of depot medroxyprogesterone acetate (DMPA) intramuscular injection has not been specifically studied. However, if an AED has been found to induce the metabolism of one specific progestin, it appears reasonable to assume that other progestins may be affected as well.

On the other hand, there are many different HC preparations available, and they may contain not only different hormones, but also different doses of EE and different doses of the same progestin. The conclusions drawn from one study investigating one $\mathrm{HC}$ preparation may not necessarily apply to another HC preparation with the same active substances but different doses. Hence, even if the available data suggest that an interaction is unlikely to occur in a specific HC-AED combination, the attending physician and the patient should take any irregular bleeding as a sign of possible contraceptive failure. 
As a consequence of the pharmacokinetic interaction between enzyme-inducing AEDs and HC, the "classic" recommendation has been to use high-dose $\mathrm{HC}$, ie, a daily EE dose of at least $50 \mu \mathrm{g} .{ }^{40-42}$ However, this advice is theoretically derived, has not been clinically proven, and has considerable conceptual weaknesses, one of them being that the ovulation-suppressing dose of EE is $\sim 100 \mu \mathrm{g}{ }^{43}$ Given the ever decreasing dose of EE in COCs, it may also be hard to find a contraceptive pill with such a high estrogen content. Moreover, despite this decades-old recommendation, a recent study from the Netherlands reported that $43.5 \%$ of WWE taking enzyme-inducing AEDs used a low dose of EE. ${ }^{44} \mathrm{~A}$ similar study performed in the UK found even a figure of $56 \%{ }^{12}$

More recent recommendations take into account the mechanism of action of modern $\mathrm{HC}$ and focus on a high progestin dose instead, since in modern HC preparations, ovulation inhibition is mediated via the progestin, not EE. ${ }^{43}$ Modern HC contains EE mainly for the purpose of creating a hormonal balance with the progestin component. Indeed, modern oral HC preparations typically contain $\sim 1.5-2$ times the ovulationinhibiting progestin dose. ${ }^{43}$ However, as enzyme induction affects not only EE but also progestins, ${ }^{45}$ even the contraceptive effect of a "high" progestin dose may be impaired by enzymeinducing AEDs, and clinical evidence for the "high progestin" concept is lacking. Consequently, neither high-dose EE nor high-dose progestin guarantees safe contraception in WWE taking enzyme-inducing AEDs, and additional contraceptive measures, eg, barrier methods, should be considered. This applies to combined (EE plus progestin) as well as progestinonly HC (oral or depot-formulations).

Hormone-releasing IUDs release a progestin and act locally on the endometrium. In contrast to systemic $\mathrm{HC}$ (oral, patch, vaginal ring, or implants), their contraceptive effect may not - at least in theory - be impaired by hepatic enzyme induction. Preliminary data from one study indeed suggest that this method is not affected by AEDs, which would make them a suitable alternative to systemic HC. ${ }^{46}$ However, this study has not been confirmed. There is also one case report on contraceptive failure with a progestin-releasing intracervical device, presumably due to simultaneous use of carbamazepine.$^{47}$ However, there are no further such reports. Nevertheless, caution is advisable until possible interactions of locally acting $\mathrm{HC}$ with enzyme-inducing AEDs have been studied more systematically.

The most obvious solution to this drug interaction problem would be to not use enzyme-inducing AEDs together with HC. With today's spectrum of available AEDs ( $>20$ in most Western countries), chances for the neurologist to avoid enzyme- inducing AEDs in fertile WWE are good. Indeed, recent surveys indicate that more and more WWE are prescribed newer, nonenzyme-inducing AEDs. ${ }^{15-18}$ In many countries however, these new AEDs may either not be available or just be too expensive. One of the "old", nonenzyme-inducing AEDs is valproate. It is very effective in a large variety of epileptic seizures and syndromes, usually well-tolerated, inexpensive, and a first-line drug for the treatment of epilepsy. It is one of the most used AEDs worldwide. Alas, it has considerable teratogenic potential and may negatively affect the cognitive outcome of children exposed in utero., ${ }^{5,6}$ This is a substantial risk, especially because half of all pregnancies in WWE occur unplanned. Moreover, typical side effects of valproate include hair loss and weight gain. Valproate may also cause polycystic ovary syndrome and metabolic disturbances. It is therefore prescribed less frequently for WWE, ${ }^{15-18}$ and the European Medicines Agency has advised physicians to not prescribe valproate to fertile women unless other treatments are ineffective or not tolerated. ${ }^{48}$ If valproate is prescribed to fertile women, highly effective contraception and adequate adherence should be ensured.

When enzyme-inducing AEDs cannot be avoided, HC should be combined with barrier methods. Recently, it has also been recommended to use $\mathrm{HC}$ in an extended-cycle pattern when enzyme-inducing AEDs are used simultaneously. ${ }^{43}$ Without the pill-free week, gonadotropin secretion and ovarian function will be continuously suppressed, which will enhance contraceptive efficacy compared to the usual pattern of use (3 weeks "on", 1 week "off"). Whether this alone provides reliable contraception despite enzyme induction remains to be proven. Until then, HC should be regarded as non-safe when combined with enzyme-inducing AEDs, and additional contraceptive methods (barrier methods) be employed.

In any case, WWE using enzyme-inducing AEDs and HC must be informed of this problem and possible solutions be presented, so that they can make an informed decision on which contraceptive method (and/or which AED) to choose. It may also be helpful to discuss this issue directly with the patient's neurologist.

\section{What HC does to AEDs}

While it has been known for over 40 years that enzymeinducing AEDs may impair the contraceptive effect of $\mathrm{HC},{ }^{49,50}$ the possibility of the opposite had practically been ignored until 2001, when it was demonstrated that COCs may reduce the serum levels of lamotrigine by $>60 \%$ and lead to loss of seizure control. ${ }^{51}$ Later studies confirmed these findings and showed that it is the estrogen component (EE) 
that is responsible for this interaction. ${ }^{52,53}$ In fact, it has been known long before 2001 that EE may affect the metabolism of quite many other drugs. ${ }^{54,55}$ Interestingly, EE has a unique dual effect on drug-metabolizing enzymes: while the activity of several CYP enzymes may be reduced, the activity of some UGTs may be increased. ${ }^{54}$ Thus, the clinical efficacy of AEDs that undergo elimination by glucuronidation may be reduced. Surprisingly, studies on the possible effects of EE on the metabolism of AEDs are still sparse.

So far, an effect of EE on the metabolism of AEDs has been demonstrated only for lamotrigine (discussed in Lamotrigine section) and, to a lesser degree, for valproate. In contrast to lamotrigine, the effect on valproate is only moderate and much less well documented (only two small studies). ${ }^{56,57}$ However, as with lamotrigine, there is large interindividual variation, and in some patients this interaction may gain clinical relevance, ie, lead to increased seizure activity. ${ }^{57}$ Oxcarbazepine and its derivative eslicarbazepine, as well as retigabine/ezogabine, are also subject to glucuronidation, but a possible effect of EE on their metabolism has not been examined so far.

It must be emphasized that, according to the current knowledge, only EE affects the metabolism of AEDs. There is no convincing data suggesting any clinically relevant effect of progestins ("mini-pill", implants, depot injections, hormonal IUDs, emergency pill) on the metabolism of lamotrigine or any other AED. In one small study, a desogestrel-only pill caused a 20\%-100\% increase in lamotrigine concentrations, but only in seven out of ten women. ${ }^{58}$ However, this study from the year 2004 still exists only in an abstract form and it has not been confirmed by others.

It should also be noted that EE is used not only in oral preparations (COCs) but also in skin patches and the vaginal ring. Accordingly, it has been found that the EE-releasing vaginal ring may reduce lamotrigine serum concentrations in a similar manner as COCs. ${ }^{52,59}$ Although not specifically studied, such an effect should also be anticipated for the EE-releasing skin patch.

\section{Lamotrigine}

Lamotrigine is metabolized by UGT enzymes that are inducible by EE. The use of EE together with lamotrigine may become challenging. First, lamotrigine concentrations fall by $\sim 50 \%-60 \%$ and seizure aggravation may occur when EE is added. ${ }^{51-53}$ This usually requires a considerable increase, and often a doubling, of the lamotrigine dose, in order to avoid seizure breakthrough. Second, lamotrigine levels rise (with considerable interindividual variation) and may even double within the pill-free week. ${ }^{53,59,60}$ This may provoke or aggravate adverse effects if the dose is not reduced during this period (Figure 1). In clinical practice though, most WWE will not experience relevant problems with side effects in the pill-free week. Finally, lamotrigine has a

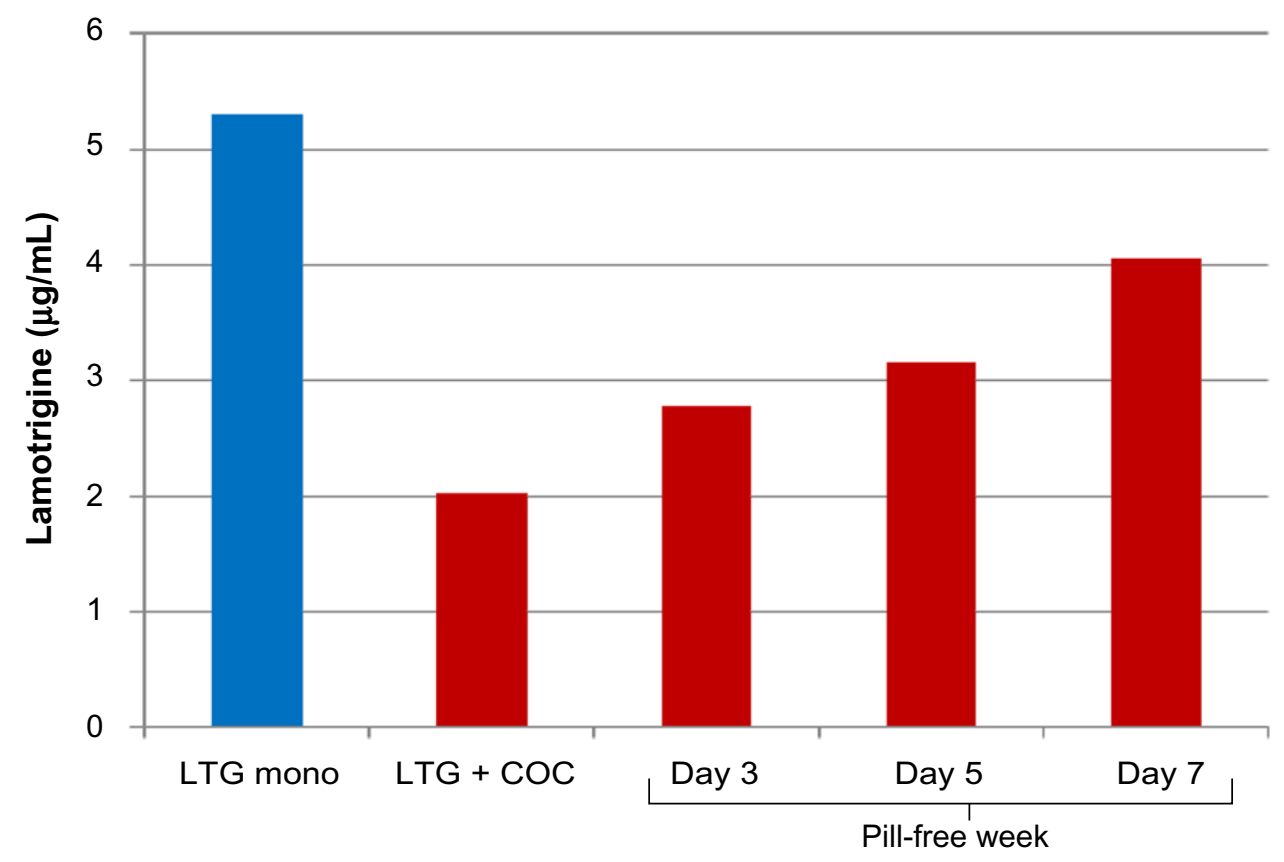

Figure I Impact of addition and withdrawal of combined oral contraception on lamotrigine (LTG) serum concentration.

Note: Permission is kindly granted by John Wiley \& Sons Ltd to adapt data from Sidhu J, Job S, Singh S, Philipson R. The pharmacokinetic and pharmacodynamic consequences of the co-administration of lamotrigine and a combined oral contraceptive in healthy female subjects. Br J Clin Pharmacol. 2006;6I (2):19I-I99.53 (c) 2005 Blackwell Publishing Ltd. Abbreviation: COC, combined oral contraceptive; mono, monotherapy. 
comparatively long half-life of 20-30 hours, which means that the pharmacokinetic steady state after dose changes or addition/removal of interacting comedication will be achieved first after $4-5$ days.

Together, all this means that COC users may have large fluctuations of the lamotrigine serum concentration during the menstrual cycle, with the possibility of both subtherapeutic and toxic levels, if the dose remains unchanged all the time. Likewise, because of the large fluctuations, serum-level measurements of lamotrigine may be considerably misleading if not always taken exactly at the same time relative to the menstrual cycle.

If active measures become necessary, dynamic dose adjustments (increase while EE is taken, decrease in the pill-free week) might prevent the loss of seizure control or the occurrence of adverse effects, but such a strategy is unrealistic as it would require great efforts from the attending neurologist and the patient, including tight clinical follow-up and regular measurement of the lamotrigine serum level over a long period of time.

All in all, the combination of lamotrigine with EE (COCs, patch, or vaginal ring) appears unfavorable. When this combination cannot be avoided, a practical solution would be to omit the pill-free week, ie, to use EE continuously. This would require only one initial dose adjustment of lamotrigine. Such "long-cycle" or "extended-cycle" regimens have been proven safe and convenient, and are becoming increasingly popular among fertile women. ${ }^{61,62}$ Recently, a COC designed for an 84-day regimen that results in bleeding only four times a year has been introduced. HC preparations for even longer cycles, 6 and 12 months, are currently being developed. ${ }^{62}$

As stated earlier, progestins apparently do not affect lamotrigine metabolism. These preparations may therefore be preferred over EE-containing $\mathrm{HC}$ when the patient uses lamotrigine. One caveat exists though. It has been found in one study that lamotrigine reduced the area under the curve of levonorgestrel by $19 \%$. This is a comparatively small effect, and the authors conclude from low progesterone serum concentrations that suppression of ovulation still was maintained..$^{53}$ No case of contraceptive failure due to lamotrigine has been published so far. However, it cannot be ruled out that in some patients, the contraceptive effect may become uncertain. Caution is therefore advisable, especially with low-dose progestin preparations (Table 1).

\section{Does HC affect epilepsy?}

The complex interaction between sexual hormones and epileptic brain activity has recently been reviewed. ${ }^{27}$ In general, neuronal excitability is increased by estrogens and reduced
Table I Pharmacokinetic interactions between $\mathrm{HC}$ and AEDs

\begin{tabular}{llll}
\hline & $\begin{array}{l}\text { AED } \\
\text { reduced } \\
\text { by EE }\end{array}$ & $\begin{array}{l}\text { EE } \\
\text { reduced } \\
\text { by AED }\end{array}$ & $\begin{array}{l}\text { Progestin } \\
\text { reduced } \\
\text { by AED }\end{array}$ \\
\hline $\begin{array}{l}\text { Old AEDs } \\
\text { Carbamazepine }\end{array}$ & NA & Yes & Yes \\
$\begin{array}{l}\text { Phenobarbital } \\
\text { Phenytoin }\end{array}$ & NA & Yes & Yes \\
Valproate & NA & Yes & Yes \\
New AEDs & Yes & No & No \\
Eslicarbazepine & NA & Yes & Yes \\
Felbamate & NA & Yes & Yes \\
Gabapentin & NA & No & No \\
Lacosamide & No & No & No \\
Lamotrigine & Yes & No & Yes \\
Levetiracetam & No & No & No \\
Oxcarbazepine & NA & Yes & Yes \\
Perampanel & NA & No & Yes \\
Pregabalin & NA & NA & NA \\
Retigabine/Ezogabine & No & No & No \\
Rufinamide & No & Yes & Yes \\
Stiripentol & NA & NA & NA \\
Topiramate & NA & Yes & No \\
Zonisamide & No & No & No \\
\hline Note:Dose & & &
\end{tabular}

Note: aDose dependent.

Abbreviations: $\mathrm{HC}$, hormonal contraception; AED, antiepileptic drug; EE, ethinyl estradiol; NA, no data available.

by progesterone. There are certain exceptions to this rule, ${ }^{27}$ but estrogens are mainly regarded as proconvulsants while progesterone is ascribed to anticonvulsant effects. This is also illustrated by the phenomenon of catamenial epilepsy, ie, epileptic seizures that occur predominantly in certain phases of the menstrual cycle. ${ }^{63}$ Neuroactive, progesteronederived steroids, such as allopregnanolone and ganaxolone, are being clinically studied for their potential as AEDs. ${ }^{64}$ So far, although $\mathrm{HC}$ has been used for $\sim 50$ years now, there is no conclusive evidence supporting the idea that $\mathrm{EE}$ or $\mathrm{HC}$ in general may aggravate epileptic seizures. ${ }^{65-67}$

\section{Osteoporosis and bone fracture risk}

It has long been known that patients taking enzyme-inducing AEDs (phenytoin, phenobarbital, carbamazepine, topiramate, and others) are at a significantly higher risk for developing osteoporosis. ${ }^{68,69} \mathrm{HC}$ has also been associated with decreased bone mineral density and increased risk of fractures. This is best documented for DMPA, particularly when used for more than 2 years. Regarding the effects of other contraceptive progestins or EE, recent meta-analyses conclude that data still are conflicting despite a large number of published studies. ${ }^{70-72}$ Some, but not all, studies show that EE may reduce bone mineral density, although this effect may depend on dose and/or treatment duration. Patients must be informed about this issue. Most importantly, the effects 
of HC (especially DMPA) and enzyme-inducing AEDs on bone mineral density may add up, which is another reason to avoid this problematic combination.

\section{Adherence}

Even the best drug treatment regimen will not work properly if the drug is not used as prescribed. Adherence to long-term therapy for chronic illnesses averages as low as 50\% in developed countries. This includes potentially life-threatening conditions such as diabetes, asthma, or epilepsy. In developing countries, the rate is even lower. ${ }^{73,74}$ Irregular drug intake is also very common among HC users. Different studies found that up to $71 \%$ of women using COCs were taking their pills irregularly. ${ }^{75,76}$ These numbers match well with population studies demonstrating that approximately half of all pregnancies occur unintended. ${ }^{9}$ Once a woman becomes pregnant, marked physiologic changes occur, including increased metabolic capacity, increased renal blood flow, and increased volume of distribution. Accordingly, it has been shown for several AEDs that their serum levels decrease by half during pregnancy, often leading to breakthrough seizures. Moreover, many patients may fear harmful effects to their unborn child and stop taking their antiepileptic medication once they discover that they are pregnant. Given the serious and potentially fatal consequences of uncontrolled epilepsy for both the mother and the unborn child, as well as the significant risk of harmful pre- and postnatal effects that several AEDs may have on the child, unplanned pregnancy in WWE should be avoided and adherence be improved.

Many factors are negatively correlated with adherence; some of them are difficult to change, such as socioeconomic status, low education, ethnicity, or co-payments. ${ }^{77}$ Apart from such factors, it may seem obvious that better patient education is a key factor to improve patient adherence. However, a large proportion of doctors lack the necessary knowledge, and many patients never receive adequate information. ${ }^{11-14,19-24}$ Also, although knowledge among doctors and patient education may have improved somewhat during recent years, still only half of WWE are able to recall the information they have been given. ${ }^{14} \mathrm{~A}$ recent study in WWE showed that an educational intervention using an informational handout led to increased knowledge on $\mathrm{HC}$ and drug interactions with AEDs, compared to standard information without the handout, although this effect was time limited. ${ }^{78}$ However, sufficient knowledge alone may not guarantee a high degree of adherence, as it has been found in a recent study that the level of knowledge was the same among adherent and nonadherent patients with epilepsy (Samsonsen C, Trondheim, Norway, unpublished data). It might therefore be speculated that many patients simply forget to take their medication. Indeed, it has been demonstrated that regular reminders such as an alarm app on the smartphone, text messages, or a digital pill dispenser improve regular pill intake significantly. ${ }^{79,80}$

Thus, in addition to purely educative measures, continuous follow-up of the patient, behavioral interventions, and systematic reminders (in the office, via telephone or mail/ e-mail, smartphone apps, or digital pill dispensers) may be useful to improve adherence and regular drug intake. ${ }^{81,82}$

\section{Practical recommendations:}

- In patients taking AEDs that interact with $\mathrm{HC}$ (phenytoin, phenobarbital, primidone, carbamazepine, topiramate, oxcarbazepine, eslicarbazepine, felbamate, rufinamide, perampanel): add other form for contraception (eg, barrier method, IUD, or a combination of them) or alternatively, discuss with the patient's neurologist if switching to a nonenzyme-inducing AED is possible.

- In patients taking lamotrigine: avoid HC that contains EE (COCs, patch, vaginal ring) or alternatively, omit EE-free week (extended-cycle regimen).

- Inform and educate patient well on: available contraceptive methods and their strengths and weaknesses; frequency and possible consequences of nonadherence; possible drug interactions between AEDs and HC; and possible pre- and postnatal adverse effects of antiepileptic medication: size and nature of teratogenic risks of AEDs and possible negative cognitive effects of AEDs.

- Advocate for long-acting nonoral contraceptive measures (IUD, implant) to avoid risk of missed pills.

- If the patient prefers oral contraception, suggest to use smartphone app (pill-reminder), digital tablet dispenser, or other measures to minimize number of missed pills.

- Schedule frequent follow-ups (in the office, via phone, or via mail/e-mail) and repeat given information (may be done by trained nurses).

- Establish regular communication with the patient's neurologist.

- Keep yourself updated on drug interactions with HC.

\section{Disclosure}

The author reports no conflicts of interest in this work.

\section{References}

1. Banerjee PN, Filippi D, Allen Hauser W. The descriptive epidemiology of epilepsy-a review. Epilepsy Res. 2009;85(1):31-45.

2. Gaffield ME, Culwell KR, Lee CR. The use of hormonal contraception among women taking anticonvulsant therapy. Contraception. 2011;83(1):16-29. 
3. Reimers A, Brodtkorb E, Sabers A. Interactions between hormonal contraception and antiepileptic drugs: clinical and mechanistic considerations. Seizure. 2015;28:66-70.

4. Tomson T, Xue H, Battino D. Major congenital malformations in children of women with epilepsy. Seizure. 2015;28:46-50.

5. Cohen MJ, Meador KJ, Browning N, et al; NEAD study group. Fetal antiepileptic drug exposure: adaptive and emotional/behavioral functioning at age 6 years. Epilepsy Behav. 2013;29(2):308-315.

6. Cummings C, Stewart M, Stevenson M, Morrow J, Nelson J. Neurodevelopment of children exposed in utero to lamotrigine, sodium valproate and carbamazepine. Arch Dis Child. 2011;96(7):643-647.

7. Fairgrieve SD, Jackson M, Jonas P, et al. Population based, prospective study of the care of women with epilepsy in pregnancy. BMJ. 2000;321(7262):674-675.

8. Davis AR, Pack AM, Kritzer J, Yoon A, Camus A. Reproductive history, sexual behavior and use of contraception in women with epilepsy. Contraception. 2008;77(6):405-409.

9. Singh S, Sedgh G, Hussain R. Unintended pregnancy: worldwide levels, trends, and outcomes. Stud Fam Plann. 2010;41(4):241-250.

10. Finer LB, Zolna MR. Unintended pregnancy in the United States incidence and disparities, 2006. Contraception. 2011;84(5):478-485.

11. Krauss GL, Brandt J, Campbell M, Plate C, Summerfield M. Antiepileptic medication and oral contraceptive interactions: a national survey of neurologists and obstetricians. Neurology. 1996;46(6):1534-1539.

12. Shorvon SD, Tallis RC, Wallace HK. Antiepileptic drugs: coprescription of proconvulsant drugs and oral contraceptives: a national study of antiepileptic drug prescribing practice. J Neurol Neurosurg Psychiatry. 2002;72(1):114-115

13. Morrell MJ, Sarto GE, Shafer PO, Borda EA, Herzog A, Callanan M. Health issues for women with epilepsy: a descriptive survey to assess knowledge and awareness among healthcare providers. JWomens Health Gend Based Med. 2000;9(9):959-965.

14. Kampman MT, Johansen SV, Stenvold H, Acharya G. Management of women with epilepsy: are guidelines being followed? Results from case-note reviews and a patient questionnaire. Epilepsia. 2005;46(8): 1286-1292.

15. Man SL, Petersen I, Thompson M, Nazareth I. Antiepileptic drugs during pregnancy in primary care: a UK population based study. PLoS One. 2012;7(12):e52339.

16. Meador KJ, Penovich P, Baker GA, et al; NEAD Study Group. Antiepileptic drug use in women of childbearing age. Epilepsy Behav. 2009;15(3):339-343.

17. Nicholas JM, Ridsdale L, Richardson MP, Ashworth M, Gulliford MC Trends in antiepileptic drug utilisation in UK primary care 1993-2008: cohort study using the General Practice Research Database. Seizure. 2012;21(6):466-470.

18. Vajda FJ, Hollingworth S, Graham J, et al. Changing patterns of antiepileptic drug use in pregnant Australian women. Acta Neurol Scand. 2010;121(2):89-93.

19. Crawford P, Hudson S. Understanding the information needs of women with epilepsy at different lifestages: results of the 'Ideal World' survey. Seizure. 2003;12(7):502-507.

20. Russell AJ, Macpherson H, Cairnie V, Brodie MJ. The care of pregnant women with epilepsy - a survey of obstetricians in Scotland. Seizure. 1996;5(4):271-277.

21. Bhakta J, Bainbridge J, Borgelt L. Teratogenic medications and concurrent contraceptive use in women of childbearing ability with epilepsy. Epilepsy Behav. 2015;52(pt A):212-217.

22. Bell GS, Nashef L, Kendall S, et al. Information recalled by women taking anti-epileptic drugs for epilepsy: a questionnaire study. Epilepsy Res. 2002;52(2):139-146.

23. Pack AM, Davis AR, Kritzer J, Yoon A, Camus A. Antiepileptic drugs: are women aware of interactions with oral contraceptives and potential teratogenicity? Epilepsy Behav. 2009;14(4):640-644.

24. Manski R, Dennis A. A mixed-methods exploration of the contraceptive experiences of female teens with epilepsy. Seizure. 2014;23(8): 629-635.
25. VerrottiA, D’Egidio C, MohnA, Coppola G, Parisi P, Chiarelli F. Antiepileptic drugs, sex hormones, and PCOS. Epilepsia. 2011;52(2):199-211.

26. Cummings LN, Giudice L, Morrell MJ. Ovulatory function in epilepsy. Epilepsia. 1995;36(4):355-359.

27. Tauboll E, Sveberg L, Svalheim S. Interactions between hormones and epilepsy. Seizure. 2015;28:3-11.

28. Isojarvi JI, Laatikainen TJ, Pakarinen AJ, Juntunen KT, Myllyla VV. Menstrual disorders in women with epilepsy receiving carbamazepine. Epilepsia. 1995;36(7):676-681.

29. Lossius MI, Tauboll E, Mowinckel P, Morkrid L, Gjerstad L. Reversible effects of antiepileptic drugs on reproductive endocrine function in men and women with epilepsy - a prospective randomized double-blind withdrawal study. Epilepsia. 2007;48(10):1875-1882.

30. Hu X, Wang J, Dong W, Fang Q, Hu L, Liu C. A meta-analysis of polycystic ovary syndrome in women taking valproate for epilepsy. Epilepsy Res. 2011;97(1-2):73-82.

31. Borthen I. Obstetrical complications in women with epilepsy. Seizure. 2015;28:32-34.

32. Lange J, Teal S, Tocce K. Decreased efficacy of an etonogestrel implant in a woman on antiepileptic medications: a case report. J Med Case Rep. 2014;8:43.

33. Schindlbeck C, Janni W, Friese K. Failure of Implanon contraception in a patient taking carbamazepin for epilepsia. Arch Gynecol Obstet. 2006;273(4):255-256.

34. Back DJ, Grimmer SF, Orme ML, Proudlove C, Mann RD, Breckenridge AM. Evaluation of Committee on Safety of Medicines yellow card reports on oral contraceptive-drug interactions with anticonvulsants and antibiotics. Br J Clin Pharmacol. 1988;25(5):527-532.

35. Zhang H, Cui D, Wang B, et al. Pharmacokinetic drug interactions involving 17alpha-ethinylestradiol: a new look at an old drug. Clin Pharmacokinet. 2007;46(2):133-157.

36. Kuhl H. Pharmacology of estrogens and progestogens: influence of different routes of administration. Climacteric. 2005;8(suppl 1):3-63.

37. Stanczyk FZ. Pharmacokinetics and potency of progestins used for hormone replacement therapy and contraception. Rev Endocr Metab Disord. 2002;3(3):211-224.

38. Rosenfeld WE, Doose DR, Walker SA, Nayak RK. Effect of topiramate on the pharmacokinetics of an oral contraceptive containing norethindrone and ethinyl estradiol in patients with epilepsy. Epilepsia. 1997;38(3):317-323.

39. Davis AR, Westhoff CL, Stanczyk FZ. Carbamazepine coadministration with an oral contraceptive: effects on steroid pharmacokinetics, ovulation, and bleeding. Epilepsia. 2011;52(2):243-247.

40. Mattson RH, Cramer JA, Darney PD, Naftolin F. Use of oral contraceptives by women with epilepsy. JAMA. 1986;256(2):238-240.

41. Crawford P, Chadwick DJ, Martin C, Tjia J, Back DJ, Orme M. The interaction of phenytoin and carbamazepine with combined oral contraceptive steroids. Br J Clin Pharmacol. 1990;30(6):892-896.

42. Practice parameter: management issues for women with epilepsy (summary statement). Report of the Quality Standards Subcommittee of the American Academy of Neurology. Neurology. 1998;51(4):944-948.

43. Schwenkhagen AM, Stodieck SR. Which contraception for women with epilepsy? Seizure. 2008;17(2):145-150.

44. Wang H, Bos JH, de Jong-van den Berg LT. Co-prescription of antiepileptic drugs and contraceptives. Contraception. 2012;85(1):28-31.

45. Haukkamaa M. Contraception by Norplant subdermal capsules is not reliable in epileptic patients on anticonvulsant treatment. Contraception. 1986;33(6):559-565.

46. Bounds W, Guillebaud J. Observational series on women using the contraceptive Mirena concurrently with anti-epileptic and other enzymeinducing drugs. J Fam Plann Reprod Health Care. 2002;28(2):78-80.

47. Ratsula K. Clinical performance of a levonorgestrel-releasing intracervical contraceptive device during the first year of use. Contraception. 1987;36(6):659-666.

48. European Medicines Agency (EMA). CMDh Agrees to Strengthen Warnings on the Use of Valproate Medicines in Women and Girls. London: European Medicines Agency (EMA); 2014. Vol EMA/709243/2014. 
49. Janz D, Schmidt D. Letter: anti-epileptic drugs and failure of oral contraceptives. Lancet. 1974;1(7866):1113.

50. Hillier K. Drug interaction with oral contraceptives. Fertil Contracept. 1978;2(1):5-8.

51. Sabers A, Buchholt JM, Uldall P, Hansen EL. Lamotrigine plasma levels reduced by oral contraceptives. Epilepsy Res. 2001;47(1-2):151-154.

52. Reimers A, Helde G, Brodtkorb E. Ethinyl estradiol, not progestogens, reduces lamotrigine serum concentrations. Epilepsia. 2005;46(9):1414-1417.

53. Sidhu J, Job S, Singh S, Philipson R. The pharmacokinetic and pharmacodynamic consequences of the co-administration of lamotrigine and a combined oral contraceptive in healthy female subjects. Br J Clin Pharmacol. 2006;61(2):191-199.

54. Shenfield GM. Oral contraceptives. Are drug interactions of clinical significance? Drug Saf. 1993;9(1):21-37.

55. Breckenridge AM, Back DJ, Orme M. Interactions between oral contraceptives and other drugs. Pharmacol Ther. 1979;7(3):617-626.

56. Galimberti CA, Mazzucchelli I, Arbasino C, Canevini MP, Fattore C, Perucca E. Increased apparent oral clearance of valproic acid during intake of combined contraceptive steroids in women with epilepsy. Epilepsia. 2006;47(9):1569-1572.

57. Herzog AG, Blum AS, Farina EL, et al. Valproate and lamotrigine level variation with menstrual cycle phase and oral contraceptive use. Neurology. 2009;72(10):911-914.

58. Schwenkhagen AM, Stodieck SRG. Interaction between lamotrigine and a progestin-only contraceptive pill containing desogestrel $75-\mu \mathrm{g}$ (Cerazette). Epilepsia. 2004;45(supp1 7):144.

59. Contin M, Albani F, Ambrosetto G, et al. Variation in lamotrigine plasma concentrations with hormonal contraceptive monthly cycles in patients with epilepsy. Epilepsia. 2006;47(9):1573-1575.

60. Christensen J, Petrenaite V, Atterman J, et al. Oral contraceptives induce lamotrigine metabolism: evidence from a double-blind, placebocontrolled trial. Epilepsia. 2007;48(3):484-489.

61. Edelman A, Micks E, Gallo MF, Jensen JT, Grimes DA. Continuous or extended cycle vs. cyclic use of combined hormonal contraceptives for contraception. Cochrane Database Syst Rev. 2014;7:CD004695.

62. Benagiano G, Carrara S, Filippi V. Safety, efficacy and patient satisfaction with continuous daily administration of levonorgestrel/ethinylestradiol oral contraceptives. Patient Prefer Adherence. 2009;3:131-143.

63. Herzog AG. Catamenial epilepsy: update on prevalence, pathophysiology and treatment from the findings of the NIH Progesterone Treatment Trial. Seizure. 2015;28:18-25.

64. Bialer M, Johannessen SI, Levy RH, Perucca E, Tomson T, White HS. Progress report on new antiepileptic drugs: a summary of the Twelfth Eilat Conference (EILAT XII). Epilepsy Res. 2015;111:85-141.

65. Dutton C, Foldvary-Schaefer N. Contraception in women with epilepsy: pharmacokinetic interactions, contraceptive options, and management. Int Rev Neurobiol. 2008;83:113-134.

66. Mattson RH, Rebar RW. Contraceptive methods for women with neurologic disorders. Am J Obstet Gynecol. 1993;168(6 pt 2):2027-2032.

67. Harden CL, Leppik I. Optimizing therapy of seizures in women who use oral contraceptives. Neurology. 2006;67(12 suppl 4):S56-S58.
68. Shen C, Chen F, Zhang Y, Guo Y, Ding M. Association between use of antiepileptic drugs and fracture risk: a systematic review and metaanalysis. Bone. 2014;64:246-253.

69. Hahn TJ. Bone complications of anticonvulsants. Drugs. 1976;12(3): 201-211.

70. Lopez LM, Chen M, Mullins Long S, Curtis KM, Helmerhorst FM. Steroidal contraceptives and bone fractures in women: evidence from observational studies. Cochrane Database Syst Rev. 2015;7:CD009849.

71. Lopez LM, Grimes DA, Schulz KF, Curtis KM, Chen M. Steroidal contraceptives: effect on bone fractures in women. Cochrane Database Syst Rev. 2014;6:CD006033.

72. World Health Organization. [webpage on the Internet]. Medical Eligibility Criteria for Contraceptive Use. 5th ed. Available from: http://www. who.int/reproductivehealth/publications/family_planning/Ex-SummMEC-5/en/. Accessed January 21, 2016.

73. World Health Organization. [webpage on the Internet]. Adherence to Long Term Therapies: Evidence for Action. Available from: http://www. who.int/chp/knowledge/publications/adherence_report/en/. Accessed January 21, 2016.

74. Samsonsen C, Reimers A, Brathen G, Helde G, Brodtkorb E. Nonadherence to treatment causing acute hospitalizations in people with epilepsy: an observational, prospective study. Epilepsia. 2014;55(11):e125-e128.

75. Aubeny E, Buhler M, Colau JC, Vicaut E, Zadikian M, Childs M. Oral contraception: patterns of non-compliance. The Coraliance study. Eur $J$ Contracept Reprod Health Care. 2002;7(3):155-161.

76. Lete I, Doval JL, Perez-Campos E, et al. Self-described impact of noncompliance among users of a combined hormonal contraceptive method. Contraception. 2008;77(4):276-282.

77. Kazerooni R, Vu K, Takizawa A, Broadhead C, Morreale AP. Association of copayment and socioeconomic status with hormonal contraceptive adherence in a female veteran population. Womens Health Issues. 2014;24(2):e237-e241.

78. Mody SK, Haunschild C, Farala JP, Honerkamp-Smith G, Hur V, Kansal L. An educational intervention on drug interactions and contraceptive options for epilepsy patients: a pilot randomized controlled trial. Contraception. 2016;93(1):77-80.

79. Wiegratz I, Elliesen J, Paoletti AM, Walzer A, Kirsch B. Adherence with ethinylestradiol $20 \mu \mathrm{g} /$ drospirenone $3 \mathrm{mg}$ in a flexible extended regimen supported by the use of a digital tablet dispenser with or without acoustic alarm: an open-label, randomized, multicenter study. Int $J$ Womens Health. 2015;7:19-29.

80. Castano PM, Bynum JY, Andres R, Lara M, Westhoff C. Effect of daily text messages on oral contraceptive continuation: a randomized controlled trial. Obstet Gynecol. 2012;119(1):14-20.

81. Halpern V, Lopez LM, Grimes DA, Stockton LL, Gallo MF. Strategies to improve adherence and acceptability of hormonal methods of contraception. Cochrane Database Syst Rev. 2013;10:CD004317.

82. Smith C, Gold J, Ngo TD, Sumpter C, Free C. Mobile phone-based interventions for improving contraception use. Cochrane Database Syst Rev. 2015;6:CD011159.
Open Access Journal of Contraception

\section{Publish your work in this journal}

Open Access Journal of Contraception is an international, peerreviewed, open access, online journal, publishing original research, reports, reviews and commentaries on all areas of contraception. In addition to clinical research, demographics and health-related aspects, the journal welcomes new findings in animal and preclinical studies
Dovepress

relating to understanding the biological mechanisms and practical development of new contraceptive agents. The manuscript management system is completely online and includes a very quick and fair peer-review system. Visit http://www.dovepress.com/testimonials.php to read real quotes from published authors. 